\title{
Automatic Patient Registration for Port Placement in Minimally Invasive Endoscopic Surgery $^{\star}$
}

\author{
Marco Feuerstein, Stephen M. Wildhirt, \\ Robert Bauernschmitt, and Nassir Navab
}

\begin{abstract}
Optimal port placement is a delicate issue in minimally invasive endoscopic surgery, particularly in robotically assisted surgery. A good choice of the instruments and endoscopes ports can avoid timeconsuming consecutive new port placement. We present a novel method to intuitively and precisely plan the port placement. The patient is registered to its pre-operative CT by just moving the endoscope around fiducials, which are attached to the patients thorax and are visible in its CT. Their 3D positions are automatically reconstructed. Without prior time-consuming segmentation, the pre-operative CT volume is directly rendered with respect to the endoscope or instruments. This enables the simulation of a camera flight through the patients interior along the instruments axes to easily validate possible ports.
\end{abstract}

\footnotetext{
* The online version of the original chapter can be found at http://dx.doi.org/10.1007/11566489_36
} 